\title{
Experiments on the Lattice Problem of Gauss
}

\author{
By H. B. Keller and J. R. Swenson
}

1. Introduction. One of the classical unsolved problems in analytic number theory is concerned with counting the number of lattice points that lie in a circle. If $A(r)$ is the number of lattice points in $D(r) \equiv\left\{(x, y) \mid x^{2}+y^{2} \leqq r^{2}\right\}$ then the problem is to find the least value of $\theta$ such that

$$
E(r) \equiv A(r)-\pi r^{2}=O\left(r^{\theta}\right) .
$$

It has been shown by Loo-Keng Hua [1] that (1) holds for $\theta=\frac{1}{2} \frac{3}{0}$ and by G. H. Hardy [2] that it does not hold for $\theta=\frac{1}{2}$. A conjecture, frequently attributed to Hardy, asserts that (1) is valid for all $\theta>\frac{1}{2}$.

The availability of high-speed digital computers suggests actually evaluating the deviation $E(r)$ for "large" values of $r$ in the hope of determining some further evidence of its behavior. At least three independent efforts in this direction have recently been made (as far as we know in the order: [3], [4], and the present paper). It is apparent from our results that the first effort [3] employed insufficiently large radii $(r<2000)$ and that the second effort [4] is incorrect for $r \geqq 3000$. The present calculations, which extend to $r=259,750$, suggest that (1) should be valid for some $\theta<\frac{1}{2} \frac{3}{0}$. However, it also seems clear that the computations which would be required to approximate any such lower estimate are impractical on an IBM 7090 or even on any other currently existing computers.

A formula for computing $A(r)$ is presented in Section 2. An efficient algorithm for evaluating this formula on a 7090 or similar machine and the corresponding program are described in Section 3. The numerical results are discussed in Section 4.

2. Formulation. For any positive real number $Z$, let $[Z]$ denote the integer part of $Z$. For any radius $r$, we define the integers

$$
K(r) \equiv[r / \sqrt{2}], \quad L(r) \equiv[r] .
$$

For all integers $i$ in $K(r)+1 \leqq i \leqq L(r)$ we define

$$
y_{i}(r) \equiv \sqrt{r^{2}-i^{2}}, \quad Y_{i}(r)=\left[y_{i}(r)\right] .
$$

If $Q(r)$ is the number of lattice points in $\left\{(x, y) \mid x>0, y>0, x^{2}+y^{2} \leqq r^{2}\right\}$ then (see Figure 1)

$$
Q(r)=K^{2}(r)+2 \sum_{i=K(r)+1}^{L(r)} Y_{i}(r) .
$$

The number of lattice points, $A(r)$, in $\mathscr{D}(r)$ can now be written as

$$
A(r)=4 Q(r)+4 L(r)+1 \text {. }
$$

The computing problem lies in the evaluation of $\sum Y_{i}$. It is relatively time

Received September 18, 1962. The work presented in this paper is supported by the AEC Computing and Applied Mathematics Center, Courant Institute of Mathematical Sciences, New York University, under contract with the U.S. Atomic Energy Commission. 


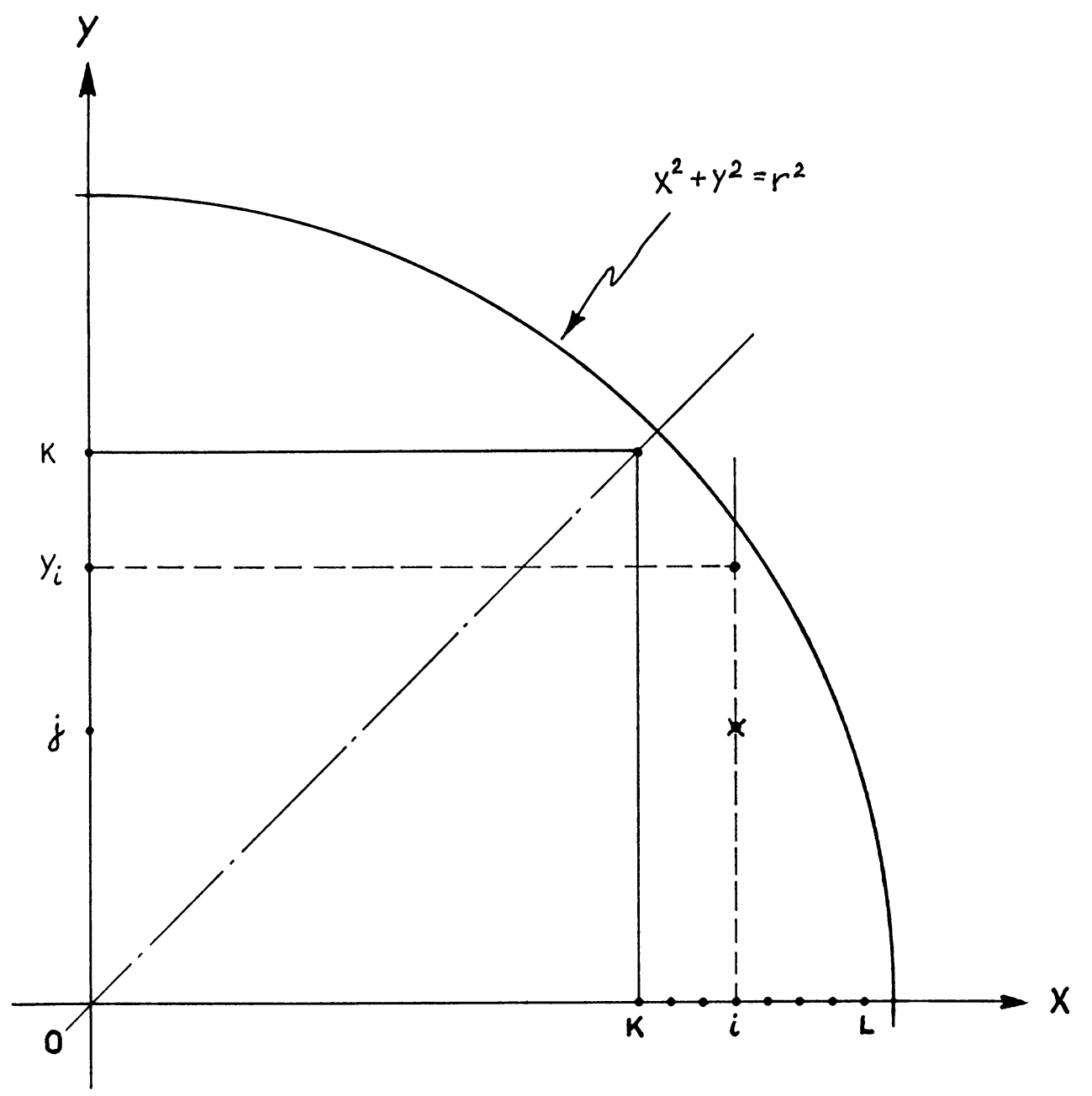

Fig. 1. Diagram to explain the counting algorithm.

consuming to determine square roots on most existing computers and the accuracy required here is to within the integral part of $y_{i}$. There is in fact no serious problem for "small" values of $r$ (say, $r<2^{13} \doteq 8.2 \times 10^{3}$ ) either with regard to time or accuracy. However, if, as we desire, $r^{2}$ is of the order of magnitude of the machine fixed point word capacity $\left(2^{36}-1\right.$ on an IBM 7090) then efficiency and extreme accuracy are crucial. We shall describe a counting procedure to evaluate $\sum Y_{i}$ which employs no square root computations and is more efficient on a 7090 than any other procedure known to us. This procedure may not be the most efficient on a machine with a very fast fixed point square root instruction.

3. Counting Algorithm and Machine Program. Let $\Im(r)$ denote the set

$$
J(r) \equiv\{(x, y) \mid y>0 ; \quad K+1 \leqq x \leqq I ; \quad(x, y) \in \mathscr{D}(r)\} .
$$

Then $\sum Y_{i}$ is just the number of lattice points contained in $J(r)$. If the lattice 
point $(i, j) \in J(r)$ and the point $(i, j+1) \notin J(r)$ then $Y_{i}(r)=j$. Furthermore, for such a lattice point $(i, j)$, if $i>K+1$ then, as a simple geometric or algebraic argument shows, $(i-1, j+1) \in J(r)$. Thus, roughly speaking, we can count the number of lattice points in $J(r)$ by tracing out a piecewise rectangular boundary which is just interior to the circular boundary of $J(r)$. This is done by starting at the point $(i, j)=(L, 0)$ and with fixed $i$ increasing $j$ until $R_{i, j}^{2} \equiv i^{2}+j^{2}>r^{2}$. When the first such $j$ is obtained and $Y_{i}(r)=(j-1)$ is accumulated in $\sum Y_{i}$ then reduce $i$ by unity. Continue this procedure until $i=K+1$. A great saving in computing time is effected by noting that

$$
R_{i, j+1}^{2}=R_{i, j}^{2}+2 j+1, \quad R_{i-1, j}^{2}=R_{i, j}^{2}-2 i+1 .
$$

Thus, on a binary machine, given $R_{L, 0}^{2}$, no multiplications are required to evaluate recursively the $R_{i, j}^{2}$.

The algorithm for computing $A(r)$ based on the above observations is described on the flow diagram, Figure 2. This algorithm was coded in FAP for the 7090 . Using fixed-point machine operations the closed loop $1.0 \rightarrow 1.1 \rightarrow 1.2 \rightarrow 1.0$ takes 19 machine cycles while the partial loop $2.0 \rightarrow 2.1 \rightarrow 2.2 \rightarrow 1.2 \rightarrow 1.0$ requires 33 cycles. The first loop must be used for each $j$ in $0<j \leqq K(r)$ or essentially $[r / \sqrt{2}] \doteq 0.7 r$ times. The partial loop is used for each $i$ in $K+1 \leqq i \leqq L$ or essentially $[r]-[r / \sqrt{2}] \doteq 0.3 r$ times. Since a 7090 machine cycle is about 2.18 microseconds, this code requires approximately $51 r \times 10^{-6}$ seconds in order to compute $A(r)$.

Using the 7090 fixed-point arithmetic operations a code based on the flow diagram of Figure 2 can compute $A(r)$ for all $r<\sqrt{2} \times 2^{17} \doteq 1.8 \times 10^{5}$. By employing the sign bit to record arithmetic data the range of $r$ is easily extended to $r<2^{18} \doteq 2.6 \times 10^{5}$. In order to do this the ordinary arithmetic machine operations must be modified. The closed loop then takes 22 cycles and the partial loop only 32 cycles. The total time to compute $A(r)$ then becomes $55 r \times 10^{-6}$ sec. The time estimates given here were found to be extremely accurate.

For values of $r$ such that $r^{2} \doteq 2^{35}$ the arithmetic in boxes 0.1 and 3.0 of the flow diagram required special higher precision techniques which do not essentially alter the above time estimates. In fact, by using certain tricks only $(Q+L)$ is required in the 7090 in order to calculate $E$ and a special 1401 output routine can be used to transform $(Q+L)$ in octal to $A(r)$ in decimal.

It is a simple matter to employ the above procedures in order to count the number of lattice points in a sphere, say of radius $\rho$. We need only compute $A\left(r_{k}\right)$ where $r_{k}=\sqrt{\rho^{2}-k^{2}}$ for $k=0,1, \cdots,[\rho]$. Using the previous estimate this requires at least $55\left(\frac{\pi}{4}\right) \rho^{2} \times 10^{-6}$ sec. for the total count.

4. Numerical Results. It is an elementary fact that $A(r)$ is a piecewise constant function with discontinuities only at values of $r$ for which $r^{2}$ can be written as the sum of the squares of two integers. Thus, for any integer $m$ there are only a finite number of distinct values of $A(r)$ for all $r \leqq m$. However, it is quite impractical, for large $m$, to compute all of these values (e.g., for $m=10^{5}$ we would require the order of $10^{10}$ computations). Hence, we content ourselves with some uniform samplings in $r$, bearing in mind the defects in any such experimental approach. 
(0)

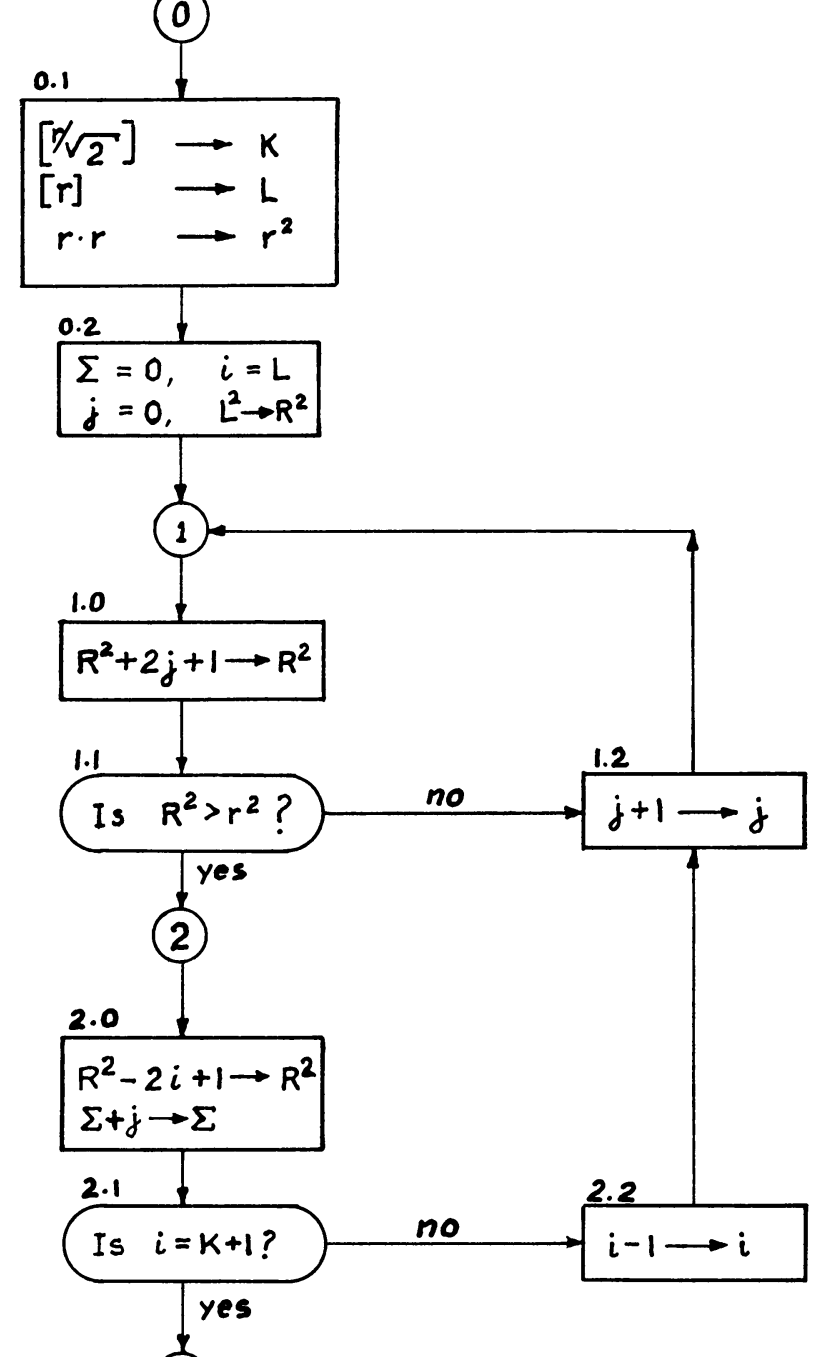

(3)

\section{0}

$$
\begin{aligned}
& K^{2}+2 \Sigma \longrightarrow Q \\
& 4(Q+L)+1 \longrightarrow A \\
& A-\pi r^{2} \longrightarrow E
\end{aligned}
$$

FIG. 2. Flow diagram for lattice point counting algorithm. 
Table 1

\begin{tabular}{|c|c|c|c|c|}
\hline$r$ & $A(r)$ & $E(r)$ & $\ln \mid \begin{array}{c}E(r) \mid / \\
\ln r\end{array}$ & $E(r) / r^{1 / 2}$ \\
\hline 250000 & $19634953910 \tilde{}$ & -.17443604 & .6005332 & -.34887201 \\
\hline 250250 & 196742432789 & -.34916004 & .656313 & -.69797101 \\
\hline 250500 & 197135722817 & -.15939204 & .593180 & -.31846601 \\
\hline 250750 & 197529403589 & -.16513204 & .595978 & -.32977001 \\
\hline 251000 & 197923477597 & -.11718104 & .568341 & -.23389401 \\
\hline 251250 & 198317943985 & -.10113704 & .556454 & -.20177101 \\
\hline 251500 & 198712801597 & -.23260204 & .623385 & -.46381501 \\
\hline 251750 & 199108054785 & -.76375403 & .533784 & -.15221901 \\
\hline 252000 & 199503696777 & -.30965604 & .646291 & -.61685001 \\
\hline 252250 & 199899736189 & -.70845903 & .527657 & -.14105801 \\
\hline 252500 & 200296165009 & -.16114304 & .593679 & -.32068701 \\
\hline 252750 & 200692987501 & -.15414904 & .590065 & -.30661601 \\
\hline 253000 & 201090202865 & -.12986204 & .576238 & -.25818101 \\
\hline 253250 & 201487810273 & -.17108404 & .598349 & -.33996701 \\
\hline 253500 & 201885812629 & .12584903 & .388575 & .24995500 \\
\hline 253750 & 202284203409 & -.23125304 & .622471 & -.45907601 \\
\hline 254000 & 202682990469 & -.11699904 & .567674 & -.23214901 \\
\hline 254250 & 203082168909 & -.13465404 & .578921 & -.26704801 \\
\hline 254500 & 203481740881 & -.69017403 & .525179 & -.13680901 \\
\hline 254750 & 203881703117 & -.24688804 & .627530 & -.48915101 \\
\hline 255000 & 204282060473 & -.18266704 & .603280 & -.36173501 \\
\hline 255250 & 204682810189 & -.15235404 & .588658 & -.30156001 \\
\hline 255500 & 205083952957 & -.86750503 & .543380 & -.17162301 \\
\hline 255750 & 205485487441 & -.11945404 & .569028 & -.23620701 \\
\hline 256000 & 205887414661 & -.14846604 & .586443 & -.29343101 \\
\hline 256250 & 206289735777 & -.57786103 & .510629 & $-.1141 \tilde{5} 401$ \\
\hline 256500 & 206692447577 & -.16861404 & .596569 & -.33292801 \\
\hline 256750 & 207095553973 & -.89750603 & .545897 & -.17712601 \\
\hline 257000 & 207499050769 & -.24079504 & .625081 & -.47498601 \\
\hline 250000.000000 & 196349539105 & -.17443604 & .600532 & -.34887201 \\
\hline .015625 & 196349565329 & -.64055402 & .334675 & -.12811000 \\
\hline .031250 & 196349589697 & -.23975003 & .440864 & -.47950000 \\
\hline .046875 & 196349614641 & .16055303 & .408603 & .32110600 \\
\hline .062500 & 196349639409 & .38485503 & .478941 & .76971000 \\
\hline .078125 & 196349664377 & .80915503 & .538729 & . 16183101 \\
\hline .093750 & 196349688785 & .67345403 & .523960 & .13469001 \\
\hline .109375 & 196349713721 & .10657504 & .560891 & .21315001 \\
\hline .125000 & 196349737825 & .62604703 & .518087 & .12520901 \\
\hline .140625 & 196349761401 & -.34165703 & .469362 & -.68331500 \\
\hline .156250 & 196349787081 & .79463503 & .537273 & .15892601 \\
\hline .171875 & 196349810921 & $.909264 \quad 02$ & .362858 & .18185200 \\
\hline .187500 & 196349835377 & .32162001 & 093988 & $.643239-02$ \\
\hline .203125 & 196349860537 & .619504 03 & .517242 & .12390001 \\
\hline .218750 & 196349885521 & .10597904 & .560439 & $.2119 \check{8} 01$ \\
\hline .234375 & 196349910209 & . 12040704 & .570709 & .24081501 \\
\hline .250000 & 196349934809 & .12603504 & .574384 & .25207101 \\
\hline .265625 & 196349959225 & .11326404 & .565788 & .22652801 \\
\hline .281250 & 196349983153 & .51692203 & .502677 & .10338401 \\
\hline .296875 & 196350007041 & -.13879803 & .396889 & -.27759600 \\
\hline .312500 & 196350031185 & -.53852003 & .505971 & -.10770401 \\
\hline
\end{tabular}


Table 1-Continued

\begin{tabular}{|c|c|c|c|c|}
\hline$r$ & $A(r)$ & $E(r)$ & $\begin{array}{c}\ln |E(r)| / \\
\ln r\end{array}$ & $E(r) / r^{1 / 2}$ \\
\hline 250000.328125 & 196350055801 & -.46624503 & .494376 & -.93248900 \\
\hline .343750 & 196350080809 & -.19706301 & .054578 & $-.394127-02$ \\
\hline .359375 & 196350105305 & -.49697702 & .314256 & $-.993954-01$ \\
\hline .375000 & 196350129513 & $\begin{array}{lll}-.385426 & 03\end{array}$ & .479060 & -.77085200 \\
\hline .390625 & 196350153921 & -.52115603 & .503334 & -.10423101 \\
\hline .406250 & 196350178761 & -.22488803 & .435715 & -.44977600 \\
\hline .421875 & 196350202577 & -.95262103 & .551862 & -.19052401 \\
\hline .437500 & 196350227629 & -.44435603 & .490507 & -.88871100 \\
\hline .453125 & 196350252989 & .37190703 & .476188 & .74381300 \\
\hline .468750 & 196350277597 & .43616903 & .489011 & .87233800 \\
\hline .484375 & 196350302053 & .348430 03 & .470941 & .69685900 \\
\hline .500000 & 196350326845 & .59668903 & .514223 & .11933701 \\
\hline
\end{tabular}

TABLE 2

\begin{tabular}{|c|c|c|c|c|}
\hline$r$ & $A(r)$ & $E(r)$ & 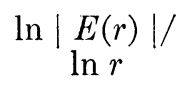 & $E(r) / r^{1 / 2}$ \\
\hline 1000 & $3141 \lesssim 49$ & -.43653502 & .546673 & -.13804401 \\
\hline 2000 & 12566345 & -.25614302 & .426680 & -.57275400 \\
\hline 3000 & 28274197 & -.13688203 & .614401 & -.24991101 \\
\hline 4000 & 50265329 & $-.153457 \quad 03$ & .606871 & -.24263701 \\
\hline 5000 & 78539677 & -.13933903 & .579641 & -.19705601 \\
\hline 6000 & 113097185 & -.15052903 & .576372 & -.19433201 \\
\hline 7000 & 153937805 & -.23502503 & .616659 & -.28090901 \\
\hline 8000 & 201061681 & -.24882903 & .613847 & -.27820001 \\
\hline 9000 & 254468477 & -.52794003 & .688522 & -.55649801 \\
\hline 10000 & $3141590 \tilde{3}$ & -.21235803 & .581767 & -.21235801 \\
\hline 20000 & 1256636857 & -.20443503 & .537210 & -.14455801 \\
\hline 30000 & 2827432965 & -.42323003 & .586666 & -.24435201 \\
\hline 40000 & 5026547529 & -.71674303 & .620453 & -.35837101 \\
\hline 50000 & 7853981045 & -.58897403 & .589511 & -.26339701 \\
\hline 60000 & 11309732881 & -.67192303 & .591718 & -.27431101 \\
\hline 70000 & 15393802989 & -.10135804 & .620392 & -.38310001 \\
\hline 80000 & 20106192121 & -.86197403 & .598702 & -.30475401 \\
\hline 90000 & 25446899381 & -.11130704 & .614932 & -.37102501 \\
\hline 100000 & 31415925457 & -.10788904 & .606596 & -.34117701 \\
\hline 125000 & 49087384401 & -.81134003 & .570777 & -.22948101 \\
\hline 150000 & 70685833345 & -.13607704 & .605434 & -.35134901 \\
\hline 175000 & 96211274253 & -.76318703 & .549801 & -.18243601 \\
\hline 200000 & 125663704421 & -.17225904 & .610481 & -.38518301 \\
\hline 225000 & 159043126541 & -.15469804 & .595922 & -.32613201 \\
\hline 250000 & 196349539105 & -.17443604 & .600532 & -.34887201 \\
\hline
\end{tabular}




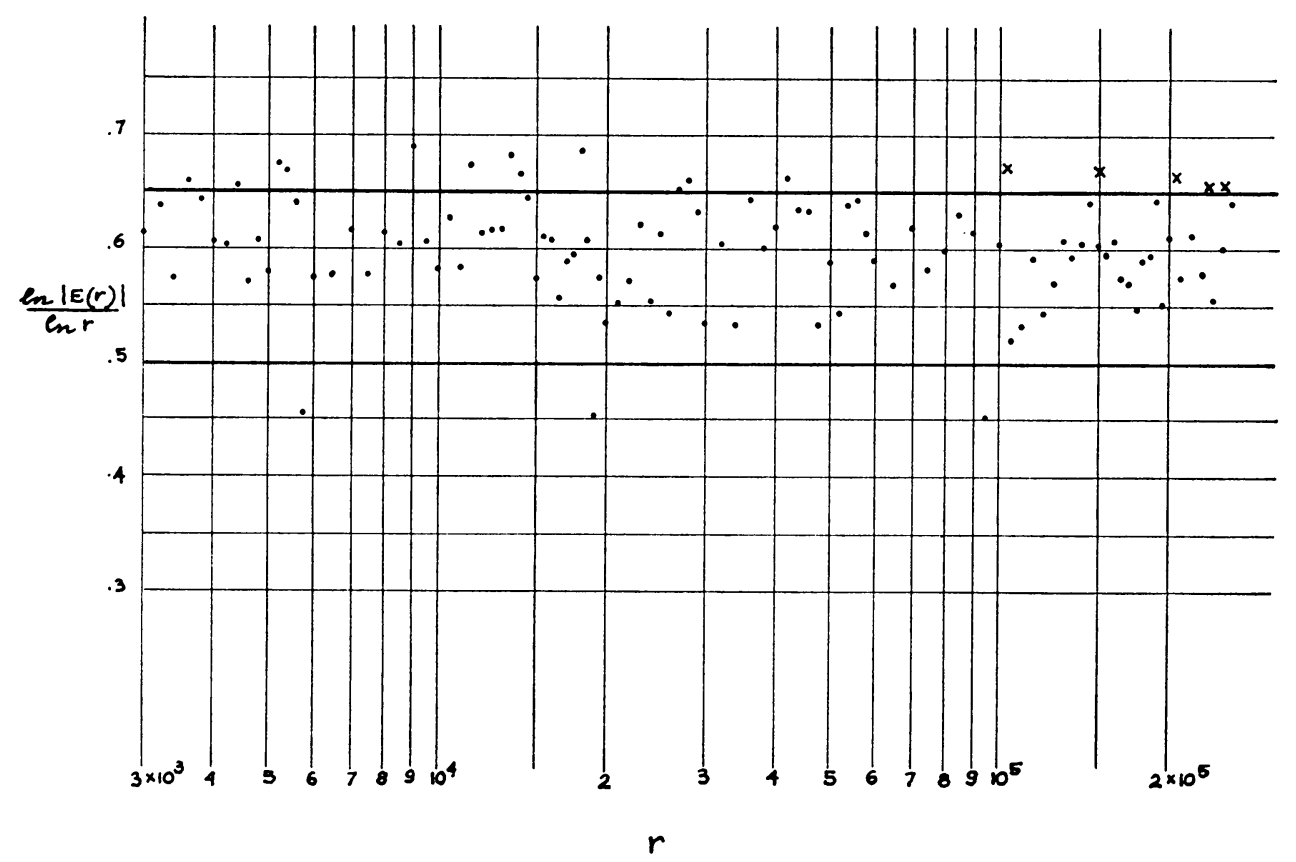

FIG. 3. Distribution of some computed values of $\ln |E(r)| / \ln r$.

Computations were made for each value of $r$ in the following sets:
a) $r=1(1) 10,000$
g) $r=200,000(1) 200,099$
b) $r=10,000(250) 100,000$
h) $r=250,000(1) 250,099$
c) $r=100,000(10,000) 150,000$
i) $r=100,000\left(\frac{1}{64}\right) 100,002$
d) $r=150,000(250) 259,750$
j) $r=150,000\left(\frac{1}{64}\right) 150,002$
e) $r=100,000(1) 100,099$
k) $r=200,000\left(\frac{1}{64}\right) 200,002$
f) $r=150,000(1) 150,099$
l) $r=250,000\left(\frac{1}{64}\right) 250,002$.

The quantities computed were: $E(r), E(r) / r^{1 / 2}, \ln |E(r)| / \ln r, \ln \left|E(r) / r^{1 / 2}\right| / \ln r$, and $E(r) /(r \ln r)^{1 / 2}$. The values of $A(r)$ and $E(r)$ were compared with all the values reported in [4]. Exact agreement for $A(r)$ and agreement to at least five digits for $E(r)$ was observed for $r=1(1) 1,000$ and $r=2,000$. However, the remaining 27 values of $A(r)$ for $r$ in $3,000 \leqq r \leqq 200,000$ reported in [4] did not agree with our values. Independent check calculations were made for $r=3000,=4000,=5000$ by using equations (2)-(5). The square roots were evaluated by means of a NewtonRaphson subroutine and exact agreement for $A(r)$ with those computed by the method of Section 3 was obtained. Thus, we conclude that the results in [4] are incorrect for $r \geqq 3000$. We also believe that our calculations are correct for all reported values of $r$. Since our method depends upon $r$ only through the quantities $r^{2}, K(r)$, and $L(r)$ and then only integer arithmetic is performed on numbers which fit into a machine word, it seems quite likely that our claim is justified.

Table 1 lists a small sample of the numerical results from sets d) and 1) and Table 2 lists sample results from sets a) $-d)$. The numbers in the columns headed $E(r)$ and $E(r) / r^{1 / 2}$ are in floating decimal notation with signed exponents and those in the $A(r)$ column are integers. Positive values of $E(r)$ were observed to be ex- 
tremely rare for integer values of $r$. For example, in the approximately 890 cases contained in sets $\mathrm{c}$ ) $-\mathrm{h}$ ) only one such value was found, namely, $r=253,500$. However, in the sets i)-l) the sign distribution of $E(r)$ was about uniform or perhaps even slightly biased in favor of positive values.

In Figure 3 values of $\ln |E(r)| / \ln r$ vs. $r$ are plotted on a semi-log scale. The dots have the abscissae: $r=3000(200) 6000(500) 2 \times 10^{4}\left(10^{3}\right) 3 \times 10^{4}\left(2 \times 10^{3}\right) 6 \times$ $10^{4}\left(\tilde{5} \times 10^{3}\right) 2 \times 10^{5}\left(10^{4}\right) 2.6 \times 10^{5}$. The horizontal lines in Figure 3 of ordinate 0.5 and 0.65 represent respectively Hardy's lower bound and Hua's upper bound on the order $\theta$ in equation (1). The points marked with an $X$ in Figure 3 were plotted to indicate that not all values computed for $r>10^{5}$ were below Hua's bound. Many such values of $\ln |E(r)| / \ln r>0.65$ were obtained in the sets i)-1) at non-integral values of $r$. The largest such value observed in $10^{5} \leqq r \leqq 259,750$ was 0.672 at $r=103,000$.

The results summarized in Figure 3 clearly suggest that ( 1 ) is valid for $\theta=0.70$ or even perhaps for $\theta=0.68$. But since it is known to be valid for all $\theta \geqq 0.65$ no useful quantitative estimates are obtained. However, an extrapolation of these data does suggest that a smaller order should suffice and that computations for larger values of $r$ could indicate this. For example, to obtain a significant improvement, say $\theta \leqq 0.60$, a crude extrapolation implies a radius of about $10^{8}$. Unfortunately, calculations for such radii, employing a partial-double-precision version of our present method on a 7090, would require at least two hours per case. Hence, they are impractical for the number of cases required to show a reasonable trend in the data. Furthermore, serious problems arise in attempting to insure the accuracy of such computations.

It was also observed that for all of our calculations $|E(r)| / r^{1 / 2}<7$. Since by Hardy's result this ratio is unbounded we must conclude that either our sampling is too crudely spaced or more likely that our range in $r$ is relatively small.

A.E.C. Computing and Applied Mathematics Center

Courant Institute of Mathematical Sciences

New York University

1. L. K. HUA, "The lattice-points in a circle," Quart. J. Math., Oxford Ser., v. 13, 1942, p. 18-29.

2. G. H. HARDY, "On the expression of a number as the sum of two squares," Quart. $J$. Math. v. 46, 1915, p. 263-283, and "On Dirichlet's divisor problem," Proc. London Math. Soc., Ser. 2 , v. 15,1916 , p. $1-25$.

3. W. Fraser \& C. C. Gothieb, "A calculation of the number of lattice points in the circle and sphere," Math. Comp., v. 16, 1962, p. 282-290.

4. H. L. MitcheLl, III, Numerical Experiments on the Number of Lattice Points in the Circle, Technical Report No. 17, Appl. Math. and Stat. Labs., Stanford University, Stanford, California. 